\title{
CUIDADO MATERNO AO RECÉM-NASCIDO NA UNIDADE DE TERAPIA INTENSIVA NEONATAL: POSSIBILIDADES E DESAFIOS ${ }^{1}$
}

\author{
THE MOTHER WHEN CARING FOR HER NEWBORN BABY AT THE \\ NEONATAL INTENSIVE CARE UNIT: POSSIBILITIES AND CHALLENGES
}

\section{CUIDADO MATERNO DEL RECIÉN NACIDO EN LA UNIDAD DE TERAPIA INTENSIVA NEONATAL: POSIBILIDADES Y DESAFÍOS}

\author{
ERIKa Da Silva DitTz \\ Roseni Rosângela de SEnA ${ }^{* *}$ \\ JoAquim ANTÔNIO CÉSAR MOTTA** \\ Elysângela DitTz Duarte ${ }^{* * * *}$
}

\begin{abstract}
RESUMO
Apresenta o resultado de um estudo qualitativo que teve como objetivo apreender os limites e as possibilidades do cuidado materno ao recém-nascido internado na Unidade de Terapia Intensiva Neonatal (UTIN). O estudo foi realizado em um hospital filantrópico em Belo Horizonte-MG e teve como participantes 11 mães de recémnascidos internados na UTIN e 11 profissionais de saúde que prestam cuidado a esses recém-nascidos. Para a coleta de dados, utilizaram-se a entrevista e a observação participante. Os dados obtidos na entrevista e os registros da observação participante foram analisados por meio da análise de discurso. Evidenciaram-se as formas de participação das mães no cuidado com os filhos e as relações e tensionamentos com os profissionais no desenvolvimento desse cuidado. Foi demonstrado que o fazer da mãe determina seu lugar no cuidado do filho e redefine suas possibilidades de ação dentro da UTIN num processo contínuo e dinâmico. Conclui-se que a participação da mãe no cuidado do filho na UTIN não é explorada em sua potencialidade, sinalizando a necessidade de ampliar os estudos a fim de solidificar as práticas já existentes e construir novas alternativas para a participação das mães.
\end{abstract}

Palavras chave: Recém-nascido, unidades de terapia intensiva neonatal, relações mãe-filho, mães.

\begin{abstract}
This paper presents the results of a qualitative study aimed to understand the limits and possibilities of maternal care to the newborn in the Intensive Neonatal Therapy Unit (NICU). The study was held at a philanthropic hospital in Belo Horizonte, M.G, and had 11 mothers of newborns hospitalized at the NICU and 11 health professionals who take care of these newborns as participants. For data collection, interviews and participant observation were used. Data analysis was made through Discourse Analysis. The data evidences the forms of participation of the mothers in the care given to their children and their relationships and tensions with the professionals in the development of the care. It was demonstrated that the attitude of the mother towards the child determines her role in the care of this child and redefines her possibilities of action inside the NICU in a
\end{abstract}

\footnotetext{
${ }^{1}$ Extraído da tese “A mãe no cuidado do recém nascido na Unidade de Terapia Intensiva Neoantal”, Faculdade de Medicina da Universidade Federal de Minas Gerais, 2009.

* Terapeuta Ocupacional do Hospital Sofia Feldman, Email: erikadittz@hotmail.com

** Professora Emérita da Escola de Enfermagem da UFMG, Email: rosenisena@uol.com.br

${ }^{* * *}$ Professor Departamento de Pediatria da Faculdade de Medicina da UFMG. Email: jacmota@ufmg.com.br

${ }^{* * * *}$ Professora Departamento de Enfermagem Materno-Infantil e Saúde Pública da Escola de Enfermagem da UFMG, Email: dittzduarte@ig.com.br
} 
continuous and dynamic process. It was concluded that the participation of the mother in the care process at the NICU is not explored in its potentiality, pointing out the need to amplify the studies in order to solidify the practices already existent and build up new alternatives for the participation of the mothers.

Key words: Newborn, intensive care units, neonatal, mother-child relations, mothers.

\section{RESUMEN}

Se presenta el resultado de un estudio cualitativo que tuvo como objetivo comprender los límites y las posibilidades del cuidado materno al recién nacido en la Unidad de Terapia Intensiva Neonatal (UTIN). El estudio se realizó en un hospital filantrópico en Belo Horizonte, M.G. y tuvo como participantes 11 madres de recién nacidos ingresados en la UTIN y 11 profesionales sanitarios que cuidan de estos recién nacidos. Para la recolección de datos se utilizó entrevistas y la observación participante. El análisis de los datos se llevó a cabo a través del Análisis del Discurso. Los datos dejan claro las formas de participación de las madres en el cuidado de los hijos y las relaciones y tensiones con los profesionales en el desarrollo de este cuidado. Fue demostrado que las formas de participación de la madre determina su lugar en el cuidado del hijo y redefine sus posibilidades de acción dentro de la UTIN en un proceso continuo y dinámico. Se concluyó que la participación de la madre en el cuidado del hijo en la UTIN no se explora en su potencialidad, apuntando la necesidad de ampliar los estudios a fin de solidificar las prácticas ya existentes y construir nuevas alternativas para la participación de las madres.

Palabras clave: Recién nacido, unidades de terapia intensiva neonatal, relaciones madre-hijo, madres.

Fecha recepción: 19/03/2010 Fecha aceptación: 16/03/2011

\section{INTRODUÇÃO}

A assistência ao recém-nascido, especialmente àqueles nascidos prematuramente, passou por profundas transformações através dos tempos. Como forma de preservar a vida dos recém-nascidos prematuros, foi criada a incubadora, pelo obstetra francês Stéphane Etienne Tarnier, em 1880 (1). As incubadoras foram projetadas com paredes de vidro para promover o envolvimento materno e facilitar que as mães vissem seus filhos (2).

Pierre Budin, discípulo de Tarnier, deu continuidade ao trabalho de seu mestre e também dispensou atenção às crianças que ajudava a nascer. Em 1907, Budin publicou o primeiro livro de neonatologia, The Nursling, com orientações para o cuidado do prematuro que incluíam controle da temperatura corporal, higiene, alimentação adequada e presença constante das mães no cuidado $(3,4)$.

Nos berçários do obstetra francês Pierre Budin, a presença da mãe era justificada pelo que constatara em suas observações: "as mães, quando separadas dos filhos pequenos, logo perderam todo o interesse naqueles que elas não puderam cuidar ou ninar" (5). Budin recomendava que a presença e a participação da mãe no cuidado com seu bebê fossem estimuladas, evitando que as crianças ficassem em orfanatos. A presença da mãe nos berçários visava, além do estabelecimento do vínculo entre mãe e filho, a estimulação do aleitamento materno (4).

No fim do século XIX e início do século XX, Martin Cooney, aluno de Budin, passa a realizar exposições com crianças prematuras em incubadoras, onde eram colocadas para crescer. Cooney participou da Exposição de Berlim em 1896 e de outras exposições na Inglaterra e nos Estados Unidos. Posteriormente se instalou em Couney Island onde cuidou, com sucesso, de mais de cinco mil prematuros (5). Cooney aumentou a consciência de que as crianças prematuras poderiam sobreviver e se desenvolver como pessoas normais (2). Em decorrência dos sucessos clínico e comercial alcançados por ele, foram criados vários centros para assistência 
ao prematuro (6).

Ao contrário de seu professor Budin, Martin Cooney não permitia a presença da mãe junto de seu filho prematuro. Nas exposições de bebês prematuros em incubadoras que realizava, as mães não pagavam ingresso para ver os seus filhos mas não podiam cuidar deles (2). Os efeitos dessa conduta foram percebidos por Cooney quando, ao terminar o período das exposições, as mães relutavam em receber os bebês que sobreviviam (5).

A preocupação com a assistência à saúde integral e humanizada surgiu no final da década de 1960. Após estudos realizados sobre os efeitos da separação do binômio mãe-filho, a prematuridade e a internação prolongada foram reconhecidas como fatores de risco para atraso no desenvolvimento, seqüelas neurológicas e maus-tratos familiares $(5,7-10)$.

Esses achados foram orientadores de mudanças na assistência ao recém-nascido, que passaram a considerar a presença dos pais junto ao filho internado, o incentivo à participação da mãe no cuidado ao filho e na tomada de decisão sobre o tratamento, a implementação de grupos de apoio aos familiares e a liberação de visitas de outros membros da família (11).

O aumento da sobrevida do prematuro traz a necessidade de outras formas de atendimento "[...] como o afeto, o vínculo, o acolhimento, o desenvolvimento integral da criança e da família, a assistência multiprofissional, o seguimento a longo prazo, a desospitalização, dentre outras" (12).

Sobre as transformações na assistência ao recém-nascido, e utilizando as reflexões acerca dos processos de mudança no meio ambiente construído, a paisagem atual é formada a partir de um processo de supressão, acumulação e superposição, mas não deixa de trazer, em si, representações de um tempo do passado. Essas representações são heranças que revelam as combinações que eram possíveis em um dado tempo e lugar (13).

Diante disso, deve-se considerar que as mudanças na assistência ao recém-nascido na Unidade de Terapia Intensiva Neonatal (UTIN) não implicam em negar o que tem sido feito. Todas as construções, conhecimento e prática ocorreram em resposta à necessidade de cuidado do recém-nascido. São as implicações desses resultados, tanto para a assistência ao recém-nascido, quanto para as relações entre mãe, recém-nascido e profissionais, que sinalizam para o constante repensar das práticas nesse espaço assistencial.

O presente estudo tem como objetivo apreender os limites e as possibilidades do cuidado materno ao recém-nascido internado na UTIN.

\section{MÉTODO}

Optou-se pela metodologia qualitativa, pelas possibilidades de uma aproximação do objeto de estudo, considerando sua subjetividade, sua complexidade e seu dinamismo.

Partiu-se do entendimento de que os fatos estão aí, mas somente se tornam históricos mediante a identificação das relações que os definem, ou seja, a historicidade dos fatos é o que permite reconhecer as categorias da realidade e de análise (13).

Nessa perspectiva, optou-se pela dialética, para apreender as contradições existentes na realidade específica de atenção à saúde, onde profissionais, mães e recém-nascidos coexistem em um determinado tempo e espaço.

Este estudo foi realizado em um hospital filantrópico, não governamental, localizado em Belo Horizonte - Minas Gerais, Brasil, que assiste a mulher e a criança, atendendo exclusivamente a clientela do Sistema Único de Saúde (SUS). O Hospital conta com 147 leitos, 75 obstétricos e 72 neonatais, sendo os neonatais divididos em terapia intensiva (41 leitos), cuidados intermediários (25 leitos) e cuidado mãe- canguru (seis leitos).

As mães têm acesso à UTIN nas 24 horas do dia. Ainda lhes são oferecidas condições 
de permanência, em período integral, em uma unidade denominada Casa de Sofias, localizada nas proximidades do hospital, com capacidade para acolher 24 mães. Na Casa são oferecidas, às mães, condições de repouso, alimentação e acompanhamento por uma equipe multiprofissional composta por psicólogo, assistente social, terapeuta ocupacional, fisioterapeuta, enfermeiro e médico.

Para a apreensão da realidade empírica, utilizaram-se a observação participante e a entrevista.

Fizeram parte da pesquisa 11 mães que participavam do cuidado a seus filhos internados na UTIN e 11 profissionais de saúde sendo enfermeiros, médicos, fisioterapeutas e técnicos de enfermagem que prestavam cuidado a esses recém-nascidos no cenário do estudo.

Os critérios de inclusão das mães foram: elas estarem junto dos filhos, em período integral ou parcial e seus filhos estarem internados por um período igual ou superior a seis dias. Foram incluídos os profissionais que estavam presentes nas situações observadas ou mencionados pelas mães nas entrevistas.

As observações foram realizadas em diferentes horários do dia e da noite e em todos os dias da semana, inclusive finais de semana e feriados, de forma a contemplar os eventos nos quais as mães estariam presentes na Unidade, como o horário das dietas dos recém-nascidos, a passagem de informações sobre o estado clínico dos neonatos e o horário de visitas.

A escolha dos eventos a serem observados estava condicionada à presença da mãe junto ao recém-nascido e a duração da observação foi determinada pelo início e término do evento escolhido. A duração das sessões de observação variou de 10 minutos a três horas e trinta minutos. Foram realizadas no período de 18 a 28 de agosto de 2008 e registradas em um diário de campo. Foram interrompidas ao serem identificadas as repetições de diálogos e comportamentos.
As entrevistas foram realizadas com as mães dos recém-nascidos internados na UTIN incluídas na observação e tiveram como pergunta disparadora Como você cuida do seu filho internado na Unidade de Terapia Intensiva Neonatal? Para a interrupção das entrevistas, utilizou-se o critério de repetição dos discursos. As entrevistas com os profissionais, tiveram como questões disparadoras Como você percebe a mãe no cuidado do recém-nascido internado na Unidade de Terapia Intensiva Neonatal? Como a mãe contribui no cuidado do recém-nascido internado na Unidade de Terapia Intensiva Neonatal?

Após o registro da observação e a transcrição das entrevistas foi realizada a substituição dos nomes dos participantes do estudo para lhes garantir o anonimato. Às mães foram atribuídos nomes de pedras preciosas; aos profissionais, nomes de cores e aos profissionais com função de coordenação, nomes de flores. Os fragmentos identificados e utilizados na análise receberam uma codificação com o nome de quem os emitiu, a identificação de mãe (M), profissional (P) e coordenador (C) e uma numeração que indica a ordem de seu aparecimento no texto (Ex.: Girassol, C-75; Jade, M-39). Procedeuse da mesma forma na utilização dos relatos da observação participante na construção do texto.

A análise dos dados foi realizada por meio da análise de discurso por permitir "compreender a língua fazendo sentido enquanto trabalho simbólico, parte do trabalho social geral, constitutivo do homem e da sua história"(14). Dessa forma, ao realizar a análise, foi considerada a relação existente entre o discurso, os sujeitos envolvidos e as situações em que se produziu o discurso.

Para a análise dos dados, os registros obtidos por meio da observação participante foram articulados com os recortes dos discursos obtidos por meio das entrevistas, tornando possível entender o contexto de produção de sentidos e explicitar outros sentidos presentes no discurso. 
A pesquisa teve início após a aprovação pela Câmara do Departamento de Pediatria, pelo Comitê de Ética em Pesquisa da Universidade Federal de Minas Gerais (COEP/UFMG) - Parecer no ETIC 679/07 e pela instituição na qual se realizou o estudo.

Os profissionais e as mães receberam informações sobre o estudo, suas dúvidas foram esclarecidas e foi-lhes garantido que sua recusa em participar ou sua desistência a qualquer momento não acarretariam prejuízo algum para a assistência oferecida a seus filhos (no caso das mães) nem para seu trabalho na Instituição (no caso dos profissionais). Os que aceitaram participar do estudo assinaram o Termo de Consentimento Livre e Esclarecido.

\section{RESULTADOS E DISCUSSÃO}

A partir do discurso dos informantes, foi possível apreender as condições que potencializam a participação da mãe no cuidado e aquelas que oferecem obstáculo a esse fazer. Observam-se aspectos das relações necessárias ao cuidado, aspectos organizacionais e gerenciais.

Em seu relato, Ametista (M-36) destaca que o livre acesso à UTIN e as condições de permanência na Instituição durante todo o período de internação foram muito importantes para sua participação no cuidado da filha.

A situação de Ametista, residente no interior do estado, e sem parentes em Belo Horizonte, assemelha-se à de outras mães que têm seus filhos internados na Instituição. Em 2008, 67\% das mães que permaneceram na Casa de Sofias eram procedentes de outras cidades do estado (15). Isso se deve à abertura institucional para o atendimento às usuárias do SUS, tornando-se uma Maternidade de referência para o estado e, especialmente, para a Região Metropolitana de Belo Horizonte.
Os relatos dos participantes revelam que, na história institucional, um dos fatores determinantes da criação de condições de permanência da mãe na Instituição reside no reconhecimento da importância da mãe junto do filho por usuários, profissionais, gestores e comunidade local. Outro aspecto está relacionado à busca pela assistência integral, expressa na missão institucional, evidenciando o compromisso de que, no cuidado ao recémnascido, sejam consideradas sua mãe e sua família. Na medida em que o Hospital tem uma política de atendimento aos usuários do SUS, recebendo gestantes e recém-nascidos de diferentes localidades, é necessário que se desenvolvam estratégias para atender às especificidades dessas mulheres com base na justificativa da necessidade de permanência da mãe junto de seu filho.

$\mathrm{Na}$ situação relatada por Ametista, fica expresso que, além do livre acesso à UTIN, outros serviços de apoio permitem atender às necessidades ampliadas de saúde que envolvem as condições de vida e os aspectos emocionais, não se limitando às ações de recuperação, reabilitação, promoção, prevenção (16).

Segundo a autora, o cuidado em saúde pode ser entendido como um fenômeno mais amplo onde a criação de serviços e de práticas cuidadoras são orientados pelas particularidades dos usuários. Isso permite inferir que, no cenário do estudo, a capacidade de responder às necessidades de cuidado do recém-nascido, de sua mãe e de sua família é potencializada na medida em que o Serviço se organiza e cria alternativas para atender às especificidades de seus usuários, como as condições para a permanência das mães junto aos recém- nascidos.

Girassol (C-78) entende que, na assistência oferecida à mãe, devem ser considerados os aspectos "medicamentosos, o social e o psíquico". Contemplar essas dimensões demanda um trabalho multiprofissional.

Essa assertiva de Girassol evidencia que a presença da mãe na Instituição e as estra- 
tégias que sustentam essa prática são orientadas pela decisão de oferecer uma atenção integral, na medida em que a Instituição se propõe a desenvolver uma prática, como descrita por Mattos (17). Tal prática tem, como princípio, oferecer resposta ao sofrimento do usuário que procura o Serviço de Saúde. Essa resposta não deve reduzir-se aos aspectos biológicos. Por meio da escuta, os profissionais devem apreender além das demandas explícitas dos usuários no que diz respeito à saúde, reduzindo os riscos de criar silenciamentos.

Nessa perspectiva, o trabalho multiprofissional, como apontado por Girassol, mostra-se necessário à construção da integralidade uma vez que, nessa atuação, tem-se a articulação de diferentes saberes e fazeres, ampliando as possibilidades de apreender as necessidades de saúde do recém-nascido, de sua mãe e de sua família.

Há que se considerar que "a presença de múltiplos saberes e práticas, de diferentes processos de trabalho numa mesma equipe, aponta para a prática da integralidade quando não se restringe às atuações individuais ou pontuais dos profissionais" (18). Os autores utilizam a metáfora de uma orquestra, onde diferentes instrumentos executam melodias distintas, mas somente com a reunião do conjunto de melodias é que se alcança a obra desejada, pois uma peça sinfônica depende da multiplicidade tímbrica dos sons, dos mais perceptíveis aos mais sutis.

Mesmo existindo um reconhecimento sobre a pertinência do exercício de uma prática multiprofissional, prevalecem, no cuidado em saúde, as práticas que colocam em um segundo plano as de caráter preventivo, educacional, psicossocial e comunicacional em favor da racionalidade biomédica, criando obstáculos para a incorporação e a integração entre os diferentes saberes e para uma atuação na perspectiva da multiprofissionalidade. $\mathrm{O}$ trabalho em equipe implica em uma mudança na lógica que orienta os processos de trabalho e deve ser reconhecido como um valor, no modelo assistencial das instituições e na prática dos profissionais dos Serviços (19).

O trabalho em equipe demanda uma atuação integralizadora e unificada dos profissionais no gerenciamento e na produção dos atos de saúde, tendo como foco as demandas e necessidades da pessoa cuidada (20). Para tanto, faz-se necessário incorporar novos dispositivos que viabilizem a construção do trabalho em equipe, aqui entendido como "mais do que diferentes categorias profissionais atuando no mesmo espaço ou no mesmo 'objeto' /alvo de intervenção: pressupõe a tessitura de um saber-fazer comum, um fazer com" (21).

Ametista (M-34) relata que, durante sua permanência na UTIN, ficava observando a técnica de enfermagem realizar os cuidados com sua filha até que perguntou se ela mesma poderia realizar a troca de fraldas. Ametista fala que, nas primeiras trocas de fraldas realizadas por ela, a técnica de enfermagem ficava próxima observando-a, "agora eles (os profissionais) já sabem que eu troco". No caso de Rubi (M-20), a iniciativa de envolvê-la no cuidado da filha partiu da técnica de enfermagem que lhe perguntou se ela gostaria de aprender a trocar as fraldas da filha.

Os discursos de Ametista e de Rubi permitiram-nos adotar, no que se refere ao processo de trabalho em saúde, a definição de dimensão cuidadora, que consiste em um espaço para além da clínica, não sendo específico de nenhum campo profissional, mas um território para a atuação de todos. Nesse espaço operam, predominantemente, as tecnologias leves e se configura como o ponto de maior interseção entre trabalhadores e usuários, com grande potencial para produzir um cuidado alicerçado nos princípios da integralidade (22). Verifica-se que, nas situações relatadas por Ametista e Rubi, as tecnologias leves foram ordenadoras da cadeia do cuidado, na medida em que houve espaço para Ametista negociar sua participação no cuidado da filha e, no caso de Rubi, a profis- 
sional não se limitou à ação rotineira e criou possibilidades para a participação da mãe no cuidado. Pelo exposto, os relatos das participantes indicam que, mesmo que o cuidado ao recém-nascido internado na UTIN seja marcado por determinismos, que visam assegurar os melhores resultados no enfrentamento dos problemas de saúde, observa-se a existência de uma margem para o alargamento da dimensão cuidadora onde o profissional pode escapar ao que foi instituído e criar possibilidades para a participação da mãe no cuidado.

Em seu relato, Cravo (C-82) considera que houve avanços na assistência ao recémnascido decorrentes da presença da mãe na UTIN. Mas as possibilidades de participação da mãe não são plenamente exploradas porque o cuidado se dá a partir do entendimento do profissional sobre como a mãe pode participar e não na perspectiva de uma construção conjunta.

No entendimento de Cinza (P-145), o profissional que acompanha o recém-nascido deve estimular a presença e a participação da mãe no cuidado do filho. Por isso, ao perceber que a mãe não tem ido à Unidade, Cinza telefona para a Casa de Sofias estimulando-a a ir à UTIN ficar com o bebê.

Em uma situação observada (Âmbar, M-59), uma profissional orienta a mãe a tocar seu bebê utilizando toda a palma da mão de forma firme e constante e, em seguida, estimula a mãe a identificar os sinais comportamentais emitidos por seu bebê ao ser tocado. Após essas orientações, a profissional mostra para a mãe que a fralda que o bebê está usando é de um tamanho maior que o indicado, dificultando o posicionamento adequado dos membros inferiores do bebê. Junto com a mãe, a profissional faz uma adaptação da fralda para adequá-la ao tamanho do bebê e a mãe se oferece para adaptar as outras fraldas que serão usadas no decorrer do dia.

O relato da situação evidencia que a intervenção realizada pela profissional acionou a possibilidade de a mãe cuidar de seu bebê. Alguns autores $(23,24)$ ajudam nessa análise, ao defenderem que a participação da mãe no cuidado deve fazer parte do plano de cuidados do recém-nascido na UTIN, não podendo ser relativizada com base na opinião de cada profissional. Em outro estudo (25) as mães expressaram que, durante a internação do filho na UTIN, seus sentimentos se alternavam entre a exclusão e a participação no cuidado, com o predomínio do sentimento de exclusão. As pesquisadoras concluíram que o sentimento de participação materna estava relacionado à existência de diálogo com o profissional que cuidava do recémnascido. A comunicação estabelecida entre os profissionais e as mães foi identificada como um fator que reduz o estresse dos pais durante a internação do filho na UTIN (26).

Como uma forma de favorecer a participação da mãe no cuidado, alguns autores $(27,28)$ defendem a inclusão da participação guiada no plano de cuidados do recémnascido internado na UTIN. A participação guiada é um processo pelo qual uma pessoa com experiência ajuda a outra pessoa com menos experiência a desenvolver habilidades para desempenhar atividades significativas da vida cotidiana. No contexto da assistência ao recém-nascido internado na UTIN, a participação guiada consiste em o profissional de saúde acompanhar a mãe enquanto ela cuida do bebê. De acordo com os autores, a participação guiada é mais do que orientar e responder a perguntas. É uma forma de envolver a mãe no cuidado e de auxiliá-la a desempenhar atribuições relacionadas à prática da maternidade.

Os dados permitem reconhecer que, mesmo que alguns relatos dos participantes deste estudo apontem intervenções favorecedoras da participação materna, essas ações não estavam incorporadas ao fazer cotidiano dos profissionais de saúde, ocorrendo em situações específicas.

Pode-se considerar que a ocorrência de situações que possibilitam a participação ma- 
terna deve-se a profissionais que Laranja ( $\mathrm{P}$ 102) descreve como aqueles que "aceitam" os familiares e aqueles que são "devotos" dos familiares. Para Laranja, os "devotos" são aqueles que se responsabilizam por negociar com o restante da equipe a possibilidade de a mãe realizar alguns cuidados com o bebê, como o cuidado mãe canguru.

$\mathrm{O}$ discurso de Cravo (C-87) evidencia a necessidade de mudanças na forma de planejar e realizar o cuidado na UTIN, com vistas a criar possibilidades para a participação da mãe. Cravo utiliza a rotina do banho dos bebês para exemplificar como a equipe poderia estabelecer acordos com a mãe e incluíla nessa atividade. Cravo relata que as mães começam a chegar à UTIN por volta das oito e meia, pois, antes, elas tomam o café da manhã, retiram o leite materno e se arrumam para ir ao Hospital. Assim, quando as mães chegam à Unidade, a maioria dos bebês já tomou o banho. No entendimento de Cravo, um melhor planejamento das atividades pelos profissionais de saúde possibilitaria que as mães atendessem às necessidades de cuidado dos filhos e estivessem presentes também nessa situação específica, por exemplo.

Depreende-se do relato de Cravo que, apesar da fragilidade da participação da mãe no cuidado e mesmo levando-se em conta as especificidades do contexto da assistência ao recém-nascido na UTIN, há possibilidades de construção dessa participação.

O relato de Cravo traz, ainda, elementos que permitem refletir sobre a disputa de interesses na produção do cuidado do recémnascido na UTIN e de caminhos possíveis para que essas disputas não se configurem, necessariamente, em conflitos. Apreendese, desse relato que, no cuidado do recémnascido na UTIN, tem-se o envolvimento de diversos atores, como técnico de enfermagem, técnico de radiologia, médico, fisioterapeuta e terapeuta ocupacional, que trazem para essa arena a disputa de concepções e interesses individuais e coletivos expressos muitas vezes na forma de realizarem proce- dimentos específicos de seu núcleo profissional e garantirem a sequência das atividades da cadeia de cuidado. Na situação do banho do recém-nascido, a participação da mãe implicaria em incluir mais um ator nessa arena de disputa, o que pode provocar situações de conflito ou possibilitar mudanças no processo de trabalho.

Emerge, do relato de Cravo, que a possibilidade de participação da mãe no banho do recém-nascido está orientada na utilização de práticas comunicativas que permitam a negociação e a pactuação entre o profissional e a mãe. Uma forma de lidar com as tensões e assimetrias na produção do cuidado é a instituição de praças de conversa, entendidas como um espaço onde os diferentes atores do cuidado possam expor seus projetos, desejos e necessidades frente a um determinado assunto que envolve o grupo (29). Dessa forma, esse espaço pode favorecer a que mães e profissionais manifestem seus interesses, matéria-prima para a negociação e a construção coletiva de alternativas, onde recémnascido e mãe também sejam protagonistas.

Para Gerânio (C-158), a participação da mãe no cuidado está diretamente relacionada a seu entendimento sobre as condições de saúde do filho. Esse achado tem suporte em estudo que evidenciou que o oferecimento contínuo e consistente de informações aos pais, pelos profissionais de saúde, possibilitou que eles passassem de "observadores passivos" a "participantes ativos" no cuidado dos filhos internados na UTIN (30).

Tomando como referência sua prática profissional, Cravo (C-90) considera que existem possibilidades para a mãe participar do cuidado do filho na UTIN, mas não são todas as mães que fazem progressos nesse sentido. Seu relato é permeado por questionamentos que buscam compreender essa situação: "será que são características pessoais dela? Será que são características dessa criança? Será que é a relação dela com o profissional?”.

Cravo (C-89) identifica, como um dos 
impedimentos para a participação da mãe no cuidado ao filho internado na UTIN, a tentativa de generalização em relação à participação da mãe no cuidado pelos profissionais de saúde. Na percepção de Cravo, a singularidade da vivência de cada mãe na UTIN nem sempre é considerada pelos profissionais de saúde. Dessa forma, se a equipe entende que uma ação da mãe no cuidado do filho inclui o banho, "é como se eu dissesse que todas as mães de recém-nascido internado na UTIN podem dar o banho naquela criança”. Cravo asinala que as generalizações, em relação à participação materna no cuidado, impedem os profissionais de vislumbrar novas possibilidades de cuidado. Esse achado vem ao encontro do estudo que constatou uma diversidade em relação às necessidades maternas do grupo estudado, sinalizando para a implementação de práticas cuidadoras que considerem a particularidade de cada mãe (31).

Os relatos de Cravo apontam que uma dimensão, a ser considerada no que se refere à participação da mãe no cuidado na UTIN, é a pluralidade das mães enquanto determinante de suas singularidades. Essa pluralidade é evidenciada pelo relato de Cravo, se consideramos que as mães estão em uma condição de igualdade na medida em que vivenciam a condição de ter um filho internado na UTIN, porém de diferença ao tomá-las como seres singulares, cada uma com sua história de vida, sentimentos e interesses. É o reconhecimento da singularidade das mães que possibilita, ao profissional de saúde, construir, com elas, uma relação de alteridade e as possibilidades de sua participação no cuidado.

\section{CONSIDERAÇÕES FINAIS}

A participação da mãe no cuidado do filho recém-nascido não está garantida apenas por sua presença. Acontece na interação estabelecida entre mãe, recém-nascido e pro- fissional de saúde. O diálogo entre mães e profissionais de saúde foi o dispositivo que mostrou potencialidade para o conhecimento e a visibilidade das possibilidades de participação materna no cuidado e para a criação conjunta de alternativas para concretizá-las.

Esse achado é indicativo do desafio a ser superado pela equipe, sendo um dos caminhos a incorporação de práticas comunicativas produtoras de diálogo e negociação, que favoreçam a redução da assimetria entre mães e profissionais e que reconheçam a subjetividade e as necessidades dos envolvidos no cuidado.

Verifica-se uma prática assistencial orientada prioritamente por normas e técnicas, reduzindo a possibilidade de construção da alteridade materna no cuidado do recémnascido na UTIN. Transformar práticas de saúde implica em uma análise cotidiana do fazer para se buscar uma sintonia entre as necessidades de cuidado e as práticas "desejáveis" para satisfazê-las. Isso implica olhar o outro, permitir ser olhado, conviver com a diversidade e o subjetivo e estar aberto a novos modos de viver a vida.

Mesmo existindo estratégias que favorecem a participação da mãe no cuidado ao filho internado na UTIN na Instituição cenário deste estudo, a participação materna não é explorada em sua potencialidade. Isso impõe a necessidade de estudos que fortaleçam as práticas já existentes e ajudem a construir novas alternativas para a participação das mães no cuidado, o que é expresso, pelas mães, como desejo e necessidade e, pelos profissionais de saúde, como possibilidade e desafio.

\section{REFERÊNCIAS}

1. Silverman WA. Incubator-baby side shows (Dr. Martin A. Couney). Pediatrics. 1979; 64(2): 127-141.

2. Davis L, Mohay H, Edwards H. Mothers' 
involvement in caring for their premature infants: an historical overview. J Adv Nurs. 2003; 42(6): 578-586.

3. Budin P. The nursling: the feeding and hygiene of premature and full-term infants. London: The Caxton Publishing Company; 1907. Hallada em: http:// www.neonatology.org/classics/nursling/ nursling.html. Acesso em 30 maio 2009.

4. Thomas LM. The changing role of parents in neonatal care: a historical review. Neonatal Netw. 2008; 27(2): 91100.

5. Klaus MH, Kennell JH. Assistência aos pais. Em: Klaus MH, Fanaroff AA. Alto risco em neonatologia. 4a ed. Rio de Janeiro: Guanabara Koogan; 1982.

6. Scochi CGS, Costa IAR, Yamanaka NMA. Evolução histórica da assistência ao recém-nascido: um panorama geral. Acta Paul Enf. 1996; 9(número especial): 96101.

7. Bowlby J. Separation anxiety. Int J Psychoanal. 1960; 41: 89-113.

8. Bowlby J. Cuidados maternos e saúde mental. 3a ed. São Paulo: Martins Fontes; 1995.

9. Klaus MH, Kennell JH. Pais/bebê: a formação do apego. Porto Alegre: Artes Médicas; 1993.

10. Bretherton I. The origins of attachment theory: John Bowlby and Mary Ainsworth. Dev Psychol. 1992; 28(5): 759-775.

11. Gaíva MAM, Scochi CGS. A participação da família no cuidado ao prematuro em UTI neonatal. Rev Bras Enferm. 2005; 58(4): 444-448.

12. Scochi CGS. A humanização da assistência hospitalar ao bebê prematuro: bases teóricas para o cuidado de enfermagem [Tese Livre Docência]. Ribeirão Preto: Escola de Enfermagem de Ribeirão Preto; 2000.

13. Santos M. Pensando o espaço do homem. 5a ed. São Paulo: USP; 2007.

14. Orlandi EP. Análise do discurso: princí- pios e procedimentos. 7a ed. Campinas, SP: Pontes; 2007.

15. Hospital Sofia Feldman. Indicadores 2008. Belo Horizonte: [s.n.]; 2008.

16. Melo EAS. Gestos de autoria: construção do sujeito da escrita na alfabetização. Comentado em: Baronas RL, org. Identidade, cultura e linguagem. Campinas (SP): Pontes Editores; 2005.

17. Mattos RA. Os sentidos da integralidade: algumas reflexões acerca de valores que merecem ser defendidos. Em: Pinheiro $R$, Mattos AR, org. Os sentidos da integralidade na atenção e no cuidado à saúde. Rio de Janeiro: UERJ, IMS, Abrasco; 2001.

18. Gomes RS, Guizardi FL, Pinheiro R. A orquestração do trabalho em saúde: um debate sobre a fragmentação das equipes. Em: Pinheiro R, Mattos RA, org. Construção social da demanda: direito à saúde, trabalho em equipe, participação e espaços públicos. Rio de Janeiro: Cepesc/UERJ, Abrasco; 2005.

19. Peduzzi M. Trabalho em equipe de saúde no horizonte normativo da integralidade, do cuidado e da democratização das relações de trabalho. Em: Pinheiro R, Mattos RA, Barros MEB, org. Trabalho em equipe sob o eixo da integralidade: valores, saberes e práticas. Rio de Janeiro: IMS/UERJ, Cepesc, Abrasco; 2007.

20. Silva KL, Sena RR. Integralidade do cuidado na saúde: indicações a partir da formação do enfermeiro. Rev Esc Enferm USP. 2008; 42(1): 48-56.

21. Bonaldi C, Gomes RS, Louzada APF, Pinheiro R. O trabalho em equipe como dispositivo de integralidade: experiências cotidianas em quatro localidades brasileiras. Em: Barros MEB, Pinheiro $\mathrm{R}$, Mattos RA. Trabalho em equipe sob o eixo da integralidade: valores, saberes e práticas. Rio de Janeiro: IMS/UERJ, Cepesc, Abrasco; 2007.

22. Merhy EE. Em busca do tempo perdido: a micropolítica do trabalho vivo em saú- 
de. Em: Merhy EE, Onocko R, org. Agir em saúde: um desafio para o público. $2^{\text {a }}$. ed. São Paulo: Hucitec; 1997.

23. Aagaard H, Hall EO. Mothers' experiences of having a preterm infant in the neonatal care unit: a meta-synthesis. J Pediatr Nurs. 2008; 23(3): e26-36.

24. Cleveland LM. Parenting in the neonatal intensive care unit. J Obstet Gynecol Neonatal Nurs. 2008; 37(6): 666-691.

25. Wigert $H$, Johansson $R$, Berg $M$, Hellström AL. Mothers' experiences of having their newborn child in a neonatal intensive care unit. Scand J Caring Sci. 2006; 20(1): 35-41.

26. Turan T, Basbakkal Z, Özbek S. Effect of nursing interventions on stressors of parents of premature infants in neonatal intensive care unit. J Clin Nurs. 2008; 17(21): 2856-2866.

27. Pridham KF. Guided participation and development of care-giving competencies for families of low birth-weight infants. J Adv Nurs. 1998; 28(5): 948-958.

28. Heermann JA, Wilson ME, Wilhelm PA. Mothers in the NICU: outsider to partner. Pediatr Nurs. 2005; 31(3): 176181.

29. Merhy EE. Público e privado: entre aparelhos, rodas e praças. Em: Aciole GG. A saúde no Brasil: cartografias do público e do privado. São Paulo, Hucitec; Sindicato dos Médicos de Campinas e Região; 2006.

30. Mok E, Leung SF. Nurses as providers of support for mothers of premature infants. J Clin Nurs. 2006; 15(6): 726-734.

31. Punthmatharith B, Buddharat U, Kamlangdee T. Comparisons of needs, need responses, and need response satisfaction of mothers of infants in neonatal intensive care units. J Pediatr Nurs. 2007; 22(6): 498-506. 